\title{
A novel five-antibody immunohistochemical test for subclassification of lung carcinoma
}

\author{
Brian Z Ring ${ }^{1}$, Robert S Seitz ${ }^{2}$, Rodney A Beck ${ }^{2}$, William J Shasteen ${ }^{2}$, Alex Soltermann ${ }^{3}$, \\ Stefanie Arbogast ${ }^{3}$, Francisco Robert ${ }^{4}$, Marshall T Schreeder ${ }^{5}$ and Douglas T Ross ${ }^{1}$ \\ ${ }^{1}$ Department of Research and Development, Applied Genomics Inc., Burlingame, CA, USA; ${ }^{2}$ Applied \\ Genomics Inc., Huntsville, AL, USA ${ }^{3}$ Universitätsspital Zürich, Zürich, Switzerland; ${ }^{4}$ University of Alabama \\ at Birmingham, Birmingham, AL, USA and ${ }^{5}$ Clearview Cancer Center, Huntsville, AL, USA
}

\begin{abstract}
Malignant epithelial lung carcinoma can be subclassified by histology into several tumor types, including adenocarcinoma and squamous cell carcinoma. The need for a uniform method of classifying lung carcinomas is growing as clinical trials reveal treatment and side effect differences associated with histological subtypes. Diagnosis is primarily performed by morphological assessment. However, the increased use of needle biopsy has diminished the amount of tissue available for interpretation. These changes in how lung carcinomas are diagnosed and treated suggest that the development of improved molecular-based classification tools could improve patient management. We used a 551-patient surgical specimen lung carcinoma retrospective cohort from a regional hospital to assess the association of a large number of proteins with histological type by immunohistochemistry. Five of these antibodies, targeting the proteins TRIM29, CEACAM5, SLC7A5, MUC1, and CK5/6, were combined into one test using a weighted algorithm trained to discriminate adenocarcinoma from squamous cell carcinoma. Antibody-based classification on $600 \mu \mathrm{M}$ tissue array cores with the fiveantibody test was compared to standard histological evaluation on surgical specimens in three independent lung carcinoma cohorts (combined population of 1111 patients). In addition, the five-antibody test was tested against the two-marker panel thyroid transcription factor-1 (TTF-1) and TP63. Both the five-antibody test and TTF-1/TP63 panel had similarly low misclassification rates on the validation cohorts compared to morphological-based diagnosis (4.1 vs $3.5 \%)$. However the percentage of patients remaining unclassifiable by TTF-1/TP63 (22\%, 95\% Cl: $20-25 \%)$ was twice that of the five-antibody test (11\%, 95\% Cl: $8-13 \%)$. The results of this study suggest the five-antibody test may have an immediate function in the clinic for helping pathologists distinguish lung carcinoma histological types. The results also suggest that if validated in prospectively defined clinical trials this classifier might identify candidates for targeted therapy that are overlooked with current diagnostic approaches.
\end{abstract}

Modern Pathology (2009) 22, 1032-1043; doi:10.1038/modpathol.2009.60; published online 8 May 2009

Keywords: histological type; lung carcinoma; bevacizumab; adenocarcinoma; squamous cell carcinoma

Malignant epithelial lung carcinoma is classified into several histological types, including adeno-, squamous cell, and large cell carcinoma. ${ }^{1}$ Large cell and adenocarcinomas are more frequently found on the periphery of the lungs, in contrast to squamous cell carcinomas that tend to be more centrally located. Squamous cell carcinomas frequently are slower growing and metastasize late. Large cell and adenocarcinomas tend to have a worse prognosis for all stages. Recent genomic studies have begun to

Correspondence: Dr BZ Ring, PhD, Department of Research and Development, Applied Genomics Inc., 863 Mitten Road, Suite 103, Burlingame, CA 94010, USA.

E-mail: bzring@applied-genomics.com

Received 25 November 2008; revised 4 February 2009; accepted 5 February 2009; published online 8 May 2009 reveal systematic gene expression differences both between and within these histological subclasses. ${ }^{2-6}$ These molecular physiological differences, detected by large-scale gene expression profiling, suggest a potential clinical utility for subclassification by clinical histological type, ${ }^{7,8}$ but several studies have failed to find a predictive association between histological type and standard cytotoxic chemotherapy efficacy, accounting for current standard treatment strategies that do not take into account lung carcinoma histological type..$^{9-11}$

Despite the established lack of utility of distinguishing adenocarcinoma from squamous cell carcinoma for the selection of traditional chemotherapeutics in the treatment of lung carcinoma, there is growing evidence that the efficacy and toxicity of some emerging targeted or combination 
treatment strategies are associated with histological type. Small molecules that inhibit the receptor tyrosine kinase EGFR (gefitinib and erlotinib) have shown a significant clinical response only in patients with tumors that show adenocarcinoma histology in several clinical trials. ${ }^{12-14}$ Therapeutic regimens using docetaxel in combination with the nucleoside analogue gemcitabine, and permetrexed in combination with cisplatin, have a higher response rate in patients with adenocarcinoma differentiation compared to other histological types of lung carcinoma. ${ }^{15,16}$ The recombinant antibody bevacizumab, targeting the VEGF protein, has been shown to be effective when used in combination with standard first-line chemotherapy; however in patients with squamous cell carcinomas, in particular the cavitating variant, it is associated with fatal pulmonary hemorrhage. It has thus been recommended for use only in lung carcinoma patients with non-squamous cell histology. ${ }^{17-19}$ These findings have generated a renewed interest in the importance of histological type in determining treatment choices in lung carcinoma patients.

Classification of lung carcinoma into histological types is traditionally carried out by using the standard histochemical staining using hematoxylin and eosin (H\&E) and a mucin stain such as Alcian blue periodic acid-Schiff recognition of glandular/ acinar, papillary, or bronchioloalveolar growth patterns as well as mucin droplets warrants for diagnosis of adenocarcinoma. ${ }^{1}$ In contrast, the presence of cytoplasmic keratinization with squamous pearl formation, unequivocal squamous-type stratification of the epithelium or intercellular bridges (desmosomes) defines squamous cell carcinoma. In addition, unclassifiable growth patterns are often found, eg solid growing tumors with very scarce mucin droplets, pseudostratification without clear desmosomes, or cytoplasmic keratinization. Clear large cell features: large and vesicular nuclei, containing prominent red macronucleoli, may also be absent. The diagnosis is also further confounded by the use of needle biopsy as opposed to surgical biopsy for later stage tumors, which provides limited material for diagnosis. Error rates may be as high as $25 \%$ when comparing diagnosis on needle biopsy specimens to those obtained on surgical specimens. ${ }^{20}$

Though there is extensive diversity in the molecular physiology of lung tumor histological types ${ }^{3}$ and markers that differentially associate with these have been previously proposed, there is currently no widely accepted molecular-based tool to help distinguish the different histological types. Two markers regularly used by the surgical pathologist as an adjunct to morphological diagnosis are the thyroid transcription factor-1 (TTF-1) that stains tumors with adeno differentiation, as opposed to TP63 that stains squamous cell carcinoma. ${ }^{21-23}$ Staining patterns of TP63 and TTF- 1 are useful both for distinguishing small cell carcinomas from squa- mous cell carcinoma ${ }^{23}$ as well as adenocarcinoma from squamous carcinomas. ${ }^{21,22}$ However the combination of these markers has a relatively low sensitivity for a particular histological type as these markers are not necessarily specific to adenocarcinoma and squamous cell carcinoma; TTF-1 expression has been reported in a minority of lung squamous cell carcinomas and p63 has likewise been noted to be expressed a minority of lung adenocarcinomas, resulting in these markers in combination often both staining a single tumor or not staining at all, and therefore failing to classify a large fraction of lung carcinoma cases. $^{24-26}$ To establish a robust classifier of lung histological subtype, we explored 105 protein markers as classifiers on a lung carcinoma cohort with 223 adenocarcinomas and 161 squamous cell carcinomas and identified a five-antibody panel strongly associated with morphologically determined histological type. We then tested the association of this classifier panel, the five-antibody test, with pathologist-determined morphological class in three independent lung cohorts and compared it to classification with TTF-1 and TP63.

\section{Materials and methods}

\section{Patients}

Four different tissue array cohorts were used in this study (Table 1).

\section{Comprehensive Cancer Institute of Huntsville}

A total of 551 stages I and II surgically treated lung cancer patients were seen at the Comprehensive Cancer Institute of Huntsville (CCIH) between 1989 and 2003.

\section{University of Alabama at Birmingham}

A total of 195 consecutive stage I-IV lung carcinoma patients were seen at University of Alabama at Birmingham (UAB) between 1995 and 1996.

\section{Invitromed}

A total of 378 lung cancer patients from a collection of hospitals, compiled by Invitromed Inc.

Universitätsspital Zürich (Zurich)

A total of 598 stage I-IV lung carcinoma patients.

\section{Morphological Diagnosis}

For the CCIH, and UAB cohorts, pathological diagnosis was extracted from the clinical record and therefore performed by multiple pathologists at the collaborating institute. The Zurich cohort diagnosis was extracted from pathology record files and further reviewed by one pathologist (AS) and a physician (SA), with doubtful and large cell carcinoma cases discarded. For the Invitromed cohort, 
Table 1 Cohort characteristics

\begin{tabular}{lrrrr}
\hline & CCIH & UAB & Invitromed & Zurich \\
\hline Total & 551 & 195 & 378 & 538 \\
$\quad$ Adenocarcinoma & 220 & 83 & 84 & 251 \\
Squamous cell carcinoma & 161 & 67 & 129 & 265 \\
Large cell carcinoma & 35 & 6 & 115 & 0 \\
Other & 32 & 31 & 18 & 22 \\
Small cell carcinoma & 6 & 0 & 32 & 0 \\
Unknown & 97 & 8 & 0 & 0 \\
Age (median) & & & & \\
& 66 & 63 & ND & 64 \\
Sex & & & & \\
Male & & & & \\
Female & 341 & ND & ND & 384 \\
& 200 & ND & ND & 154 \\
Stage & & & & \\
1 & & & & \\
2 & 415 & 42 & ND & 120 \\
3 & 126 & 18 & ND & 111 \\
4 & 0 & 69 & ND & 48 \\
Tobacco use & 0 & 56 & ND & 15 \\
Current or ever & & & & \\
Never & 231 & 162 & ND & ND \\
Unknown & 41 & 19 & ND & ND \\
& 280 & 14 & ND & ND \\
\hline Abrevion: & & & & \\
\hline
\end{tabular}

Abbreviations: CCIH, Comprehensive Cancer Center of Huntsville; $\mathrm{ND}$, not determined; UAB, University of Alabama at Birmingham. The discovery cohort was assembled from patients seen at the CCIH. The three validation cohorts were assembled from patients at the UAB, the University Hospital Zürich (Zurich), and patients from a collection of hospitals (Invitromed). Histological type classification is from a pathological diagnosis without the aid of molecular markers.

diagnosis was performed by a single pathologist (WS) on whole section H\&Es.

\section{Antibody Scoring}

Scoring was performed on tissue arrays on a semiquantitative scale, where the lung cancer tissue present in each core was scored as negative, weak, or strong. Differentiation of weak or strong staining was based on the relative intensity of staining between cases. To ensure that the derived diagnostic model was reasonably insensitive to scoring variability, scores of weak and strong were combined into a single score of positive staining for the purpose of entering results into the weighted algorithm. Consensus scores between duplicates were derived by using single scores when duplicates were unavailable, converting a score to weak when one of the duplicates was scored as weak positive whereas the other was scored as strong positive or negative, and scoring as no information when duplicate cores were contradictory (eg, negative stain and strong stain). The antibodies used in the five- and twoantibody tests were scored as follows:

CEACAM5: scored positive when cytoplasm and/ or membrane staining was present on greater than $10 \%$ of invasive tumor cells.
SLC7A5: scored positive when plasma membrane staining was present on greater than $10 \%$ of invasive tumor cells.

TRIM29: scored positive when cytoplasm staining was present on greater than $10 \%$ of invasive tumor cells including cases where staining was predominantly basally located.

CK5/6: scored positive when cytoplasm staining was present on greater than $10 \%$ of invasive tumor cells.

MUC1: scored positive when staining was present on greater than $10 \%$ of invasive tumor cells including those where stain appeared to be mostly secreted.

TP63: scored positive when staining was present on greater than $10 \%$ of the nuclei of invasive tumor cells.

TTF-1: scored positive when staining was present on greater than $1 \%$ of the nuclei of invasive tumor cells.

\section{Immunohistochemistry}

Slides for immunohistochemistry were prepared and processed as described in previous studies. ${ }^{27}$ For each antibody, dilutions were first tested on a small 'titer' tissue array that had lung cancer cases with positive and negative cases for all antibodies in the panel, in addition to a set of tumor-derived cell lines suspended in paraffin. For the training cohort (CCIH), commercially available antibodies used in the discovery of the minimal antibody set (ER, PR, EGFR, ERBB2, KIT, CK5/6, CK17, TP53, and KI67) slides were stained by a commercial service (US Labs Inc., CA, USA).

\section{Antisera Generation and Screening}

Genes targeted for antisera generation were selected on the basis of gene expression patterns in diverse tumors as well as selected cell biological experiments. Antibodies were produced as previously described. ${ }^{27}$ Novel antisera $(n=737)$ were screened by an iterative process that entailed first determining a concentration that discriminated tissue and/or cell types on 'titer' arrays comprising an assortment of tumor and normal tissues. Antibodies determined to have an interpretable staining pattern $(\sim 400)$ were placed on tumor screening arrays comprising lung tumor specimens (no clinical follow-up available) to identify those that distinguished a significant subset of cases. Images of each stained core were obtained using an automated scanning microscope with hardware and software designed for tissue array image archiving (Bacus Laboratories, IL, USA). Data sets and images were managed in a custom Oracle-based database designed to retrieve and assemble data sets and images based on clinical annotations and other data parameters. 


\section{Model Building for Histological Type Determination}

Of the 400 antibodies screened on lung tumor tissues, 105 were selected to be stained and scored on the CCIH training cohort. $k$-Means and hierarchical clustering were used as an exploratory classification tool. Data were filtered to include only samples with expression data for at least $75 \%$ of assessed proteins and six clusters were used as an optimal classifier. A model able to discriminate adenocarcinoma from squamous cell carcinoma was developed on the same tumor sample set. For a patient to be included in the model building process, data were required to be present for at least $80 \%$ of the antisera. $\chi^{2}$-Analysis was used to identify markers with a significant association for discriminating adenocarcinoma from squamous cell carcinoma histology. Logistic regression models were additively built using these markers by iteratively comparing all models when adding one antibody at a time, choosing the best model using Mallow's Cp statistic. ${ }^{28}$ In a reverse manner a 'step-down' logistic model was derived and found to be very similar. In addition, recursive tree models were also created using all markers to search for evidence of potential expression-defined subclassifications. This did not appear to be a significant requirement for robust classification (data not shown). A linear regression model was selected that used SLC7A5, CEACAM5, MUC1, CK5/6, and TRIM29, similar to the antibodies nominated by the clustering of the gene expression patterns. This model was defined as $(1.25 \times$ SLC7A5) $-(1.43 \times$ CEACAM5 $)+$ $(2.2 \times$ TRIM29) $-(1.07 \times$ MUC1 $)+(2.27 \times$ CK5/6) -0.95 , in which each antibody is given a value of 0 or 1 based on the staining assessment. Patients are classified into the categories of adenocarcinoma and squamous cell carcinoma such that any patient with a score less than -0.95 is called adenocarcinoma by this test, greater than 0.30 is called squamous cell histological type, and patients with a score between these two values inclusive are of a undeterminable subclass and thus unclassified.

\section{Results}

\section{Training Cohort}

As part of a large project aimed at identifying novel immunohistochemical classifiers of lung carcinoma, 17 selected commercial and 88 novel antisera targeted for production by gene expression patterns (total 105) were used to stain the CCIH lung carcinoma tissue array cohort. Figure 1 shows a heat map representation of the antibody staining on 550 patients organizing patients into six clusters by $k$-means clustering. This unsupervised classification based on protein expression patterns sorted patients into groups that can be largely defined by histological class as determined by morphology at diagnosis. Cluster 1 is strongly enriched for patient samples with squamous cell carcinoma histology, which are typified by staining with antibodyrecognizing cystatin A, SLC7A5, ABCG2, NCSTN, CK5/6, TP63, and TRIM29. Clusters 2, 3, 4, and 5 are heavily populated by samples diagnosed as adenocarcinomas and are typified by staining with CEACAM5, TTF-1, MUC1, and ERBB2. The sixth cluster contains the majority of the large cell tumors but also includes a significant fraction either deemed adeno- or squamous histology at diagnosis. This cluster is typified by absence of staining with CEACAM5, CK5/6, TRIM29, and MUC1 and other markers that characterize the adenocarcinoma and squamous carcinoma clusters. These results demonstrate that the antibody staining patterns broadly reflect histological diagnosis, suggesting that the molecular features detected by these markers could form the basis of a robust diagnostic tool to aid the pathologist in distinguishing differentiation-based subtypes.

This staining data set was used to derive a linear regression model that combines results for selected squamous markers (SLC7A5, CK5/6, TRIM29) with adenocarcinoma markers (MUC1, CEACAM5) into a weighted classifier for distinguishing adenocarcinoma from squamous cell carcinoma. Samples that the model cannot unambiguously assign into one of these two subclasses are termed 'unclassifiable'. In the CCIH training cohort, the misclassification rate of the five-antibody test was low: $3 \%$ of samples designated as adenocarcinomas by morphological diagnosis were classified as squamous cell, $3 \%$ of squamous cell as designated by morphological diagnosis were classified as adenocarcinoma, and $12 \%$ of patients were unclassifiable (Table 2). Representative lung carcinoma cases are shown in Figure 2, with examples of staining with the antisera that comprise the five-antibody test.

\section{Validation in Independent Cohorts}

To test the association between histological type assignment by the five-antibody test and histological assessment by morphology, three independent lung carcinoma tissue array cohorts comprising 1111 samples in total, assembled at separate institutions (The Cleveland Clinic Foundation, Universitätsspital Zürich, and Invitromed), were stained with the five-antibody test (Figure 3). Antibody scoring rules, regression model terms, and criteria for categorization as 'adenocarcinoma', 'unclassifiable' (scoring in between the adenocarcinoma or squamous cell categorizations), and 'squamous cell carcinoma' were prospectively defined before staining and scoring. For a patient to be included for model validation, staining data were required to be present for sufficient biomarkers in the model for an unambiguous classification.

In these three independent cohorts, the classification of tumors by the five-antibody test was strongly 


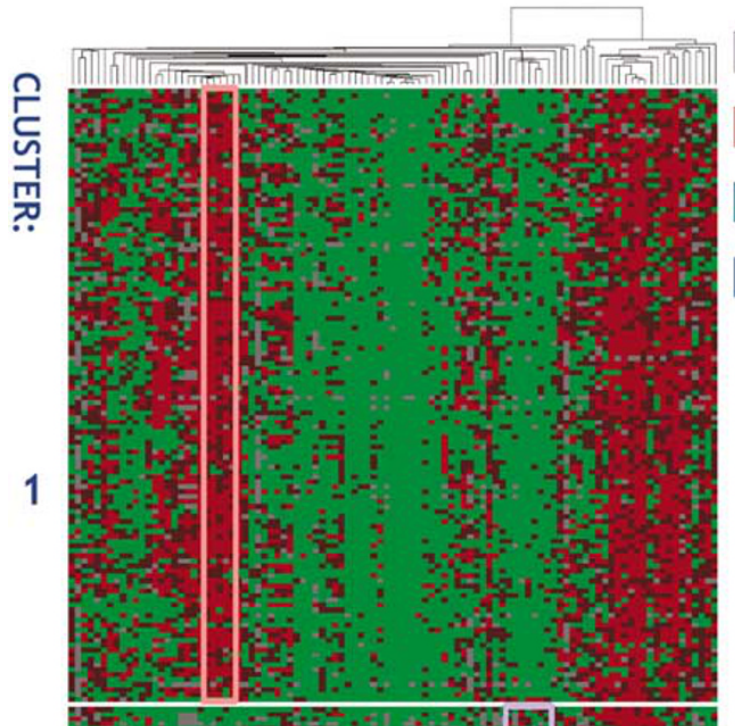

\section{Pathologist Diagnosis} Adenocarcinoma

Squamous Cell

$\square$ Large Cell

Other

$\begin{array}{llllll}0 & 25 & 50 & 75 & 100 & 125\end{array}$

2
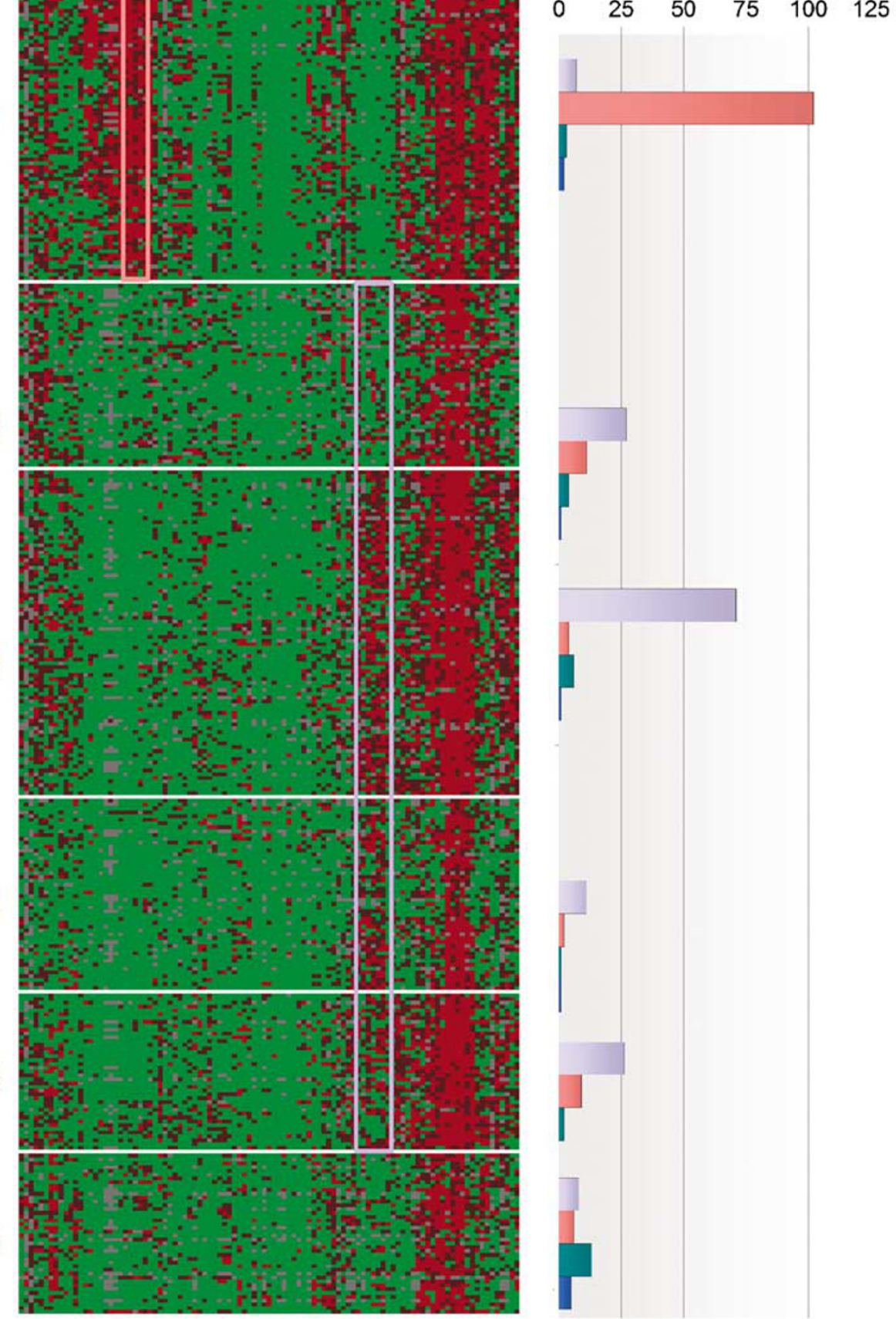

Figure 1 A heat map representation of a $k$-means clustering of antibody staining in the CCIH lung carcinoma cohort. Antibody staining is represented as positive (red) or negative (green), where the antibodies ( $x$ axis) were first hierarchically clustered, followed by $k$-means clustering of the tumor samples ( $y$ axis) into six clusters. These classes are compared to the number of patients in the different histological type diagnoses. Clusters 1 and 2 are strongly associated with morphological squamous cell carcinoma, typified by staining of CSTA, SLC7A5, ABCG2, TP63, NCSTN, CK5/6, and TRIM29 (red box). The clusters 3, 4, and 5 are heavily populated by adenocarcinomas, and are typified by staining with CEACAM5, TTF-1, MUC1, and ERBB2 (blue box). The sixth cluster contains the majority of the large cell tumors. This cluster is typified by absence of staining with CEACAM5, CK5/6, TRIM29, and MUC1. 
Table 2 Classification of tumor samples by the antibody tests

\begin{tabular}{|c|c|c|c|c|c|c|}
\hline \multirow[t]{2}{*}{ Pathologist diagnosis } & \multicolumn{3}{|c|}{ Five antibody test } & \multicolumn{3}{|c|}{$T T F-1 / T P 63$} \\
\hline & Adenocarcinoma & Unclassifiable & Squamous & Adenocarcinoma & Unclassifiable & Squamous \\
\hline \multicolumn{7}{|l|}{ CCIH } \\
\hline Adenocarcinoma & 150 & 25 & 6 & 61 & 40 & 6 \\
\hline Squamous & 5 & 15 & 126 & 5 & 18 & 71 \\
\hline \multicolumn{7}{|l|}{$U A B$} \\
\hline Adenocarcinoma & 54 & 6 & 3 & 34 & 31 & 4 \\
\hline Squamous & 2 & 1 & 42 & 1 & 12 & 34 \\
\hline \multicolumn{7}{|l|}{ Invitromed } \\
\hline Adenocarcinoma & 66 & 19 & 6 & 40 & 42 & 5 \\
\hline Squamous & 7 & 11 & 142 & 4 & 19 & 123 \\
\hline \multicolumn{7}{|l|}{ Zurich } \\
\hline Adenocarcinoma & 208 & 20 & 4 & 189 & 53 & 4 \\
\hline Squamous & 13 & 31 & 209 & 8 & 21 & 244 \\
\hline
\end{tabular}

Abbreviations: CCIH, Comprehensive Cancer Center of Huntsville; UAB, University of Alabama at Birmingham.

The tumor samples from the four cohorts that were classified as either squamous cell or adenocarcinoma by pathological diagnosis are categorized by rows, and the number of each classified by the two tests as 'squamous cell', 'unclassifiable', or 'adenocarcinoma' is exhibited.

associated with pathologist morphological assessment $\left(\chi^{2}\right.$-test $P$-value $\left.<0.0001\right)$. For tumors for which a pathologist was able to assign a histological diagnosis of adenocarcinoma or squamous cell carcinoma, the five-antibody test had a misclassification rate ranging from 4 to $5 \%$, with $4.1 \%$ (95\% CI: $2.8-5.5 \%)$ in the combined validation population (Table 2). The percentage of cases unable to be classified ranged from 6 to $12 \%$ on the validation cohorts (11\% unclassified (95\% CI: $8-13 \%$ ) in the combined validation cohorts).

\section{Comparison to TTF-1 and TP63}

Two protein markers that are often used as an aid to morphology in determining histological type are TTF-1 and TP63. A staining pattern of TP63-/ TTF-1 + has been used to help discriminate adenocarcinoma (and small cell carcinoma) from squamous cell carcinoma, which tend to stain TP63 + / TTF-1-. Similar to the five-antibody test, in the four cohorts studied herein, staining and classification by TTF-1/TP63 were confirmed to have a low misclassification rate, ranging from 2 to $5 \%$, with $3.5 \%$ misclassified (95\% CI: $2.4-4.6 \%$ ) in the combined population (Table 2; Figure 3). Defining the morphological H\&E/AB-PAS-based interpretation as the gold standard and considering only those cases where a pathologist's diagnosis was available, the two tests showed very strong concordance (Cohen's $\kappa$ coefficient of $0.972 \quad(95 \%$ CI: 0.950.99)), which reflects their low misclassification rate. However, there was a very strong difference between the five-antibody test and the TTF-1/TP63 test in the fraction of cases for which the tests were informative. The percentage of cases unable to be classified by TTF-1/TP63 (either TP63+/TTF-1+ or TP63-/TTF-1-) ranged from 14 to $37 \%$ on the validation cohorts, and $22 \%$ (95\% CI: $20-25 \%$ ) in the combined cohorts, twice that found using the five-antibody test. The larger fraction of cases interpretable by the five-antibody test results in a significantly higher overall rate of correct histological type assignment $(P<0.0001$ for the difference in the proportion classifiable between the two tests).

Clinical trials in support of the VEGF-targeted therapeutic antibody, bevacizumab, as well as studies exploring EGFR inhibitors have created an increased need to distinguish adenocarcinomas from squamous cell carcinoma. To qualify for treatment with bevacizumab a pathologist is required to affirm a diagnosis of adenocarcinoma differentiation, which effectively groups together unclassifiable and squamous cell cases as poor candidates for bevacizumab treatment. In these cohorts, the five-antibody test was more sensitive for identifying adenocarcinoma cases compared to TTF-1/TP63, with a sensitivity of $88.6 \% \quad(95 \%$ CI: $85.9-91.3 \%)$, compared to $74.6 \% \quad(95 \%$ CI: $71.4-77.7 \%$ ). Similarly, the NPV of the five-antibody test was superior, $84.8 \%$ (95\% CI: $81.2-88.3 \%$ ) compared to $63.7 \%$ (95\% CI: $59.5-67.8 \%)$ for TTF-1/TP63. This difference was primarily due to the lower rate of unclassified samples by the fiveantibody test (Figure 4). Specificity and PPV were insignificantly different between the two tests (data not shown).

\section{Large Cell Carcinoma Classification}

Morphological large cell carcinoma is largely a diagnosis of exclusion of features characteristic of adenocarcinoma or squamous cell carcinoma. Among lung carcinoma cases for which both tests 


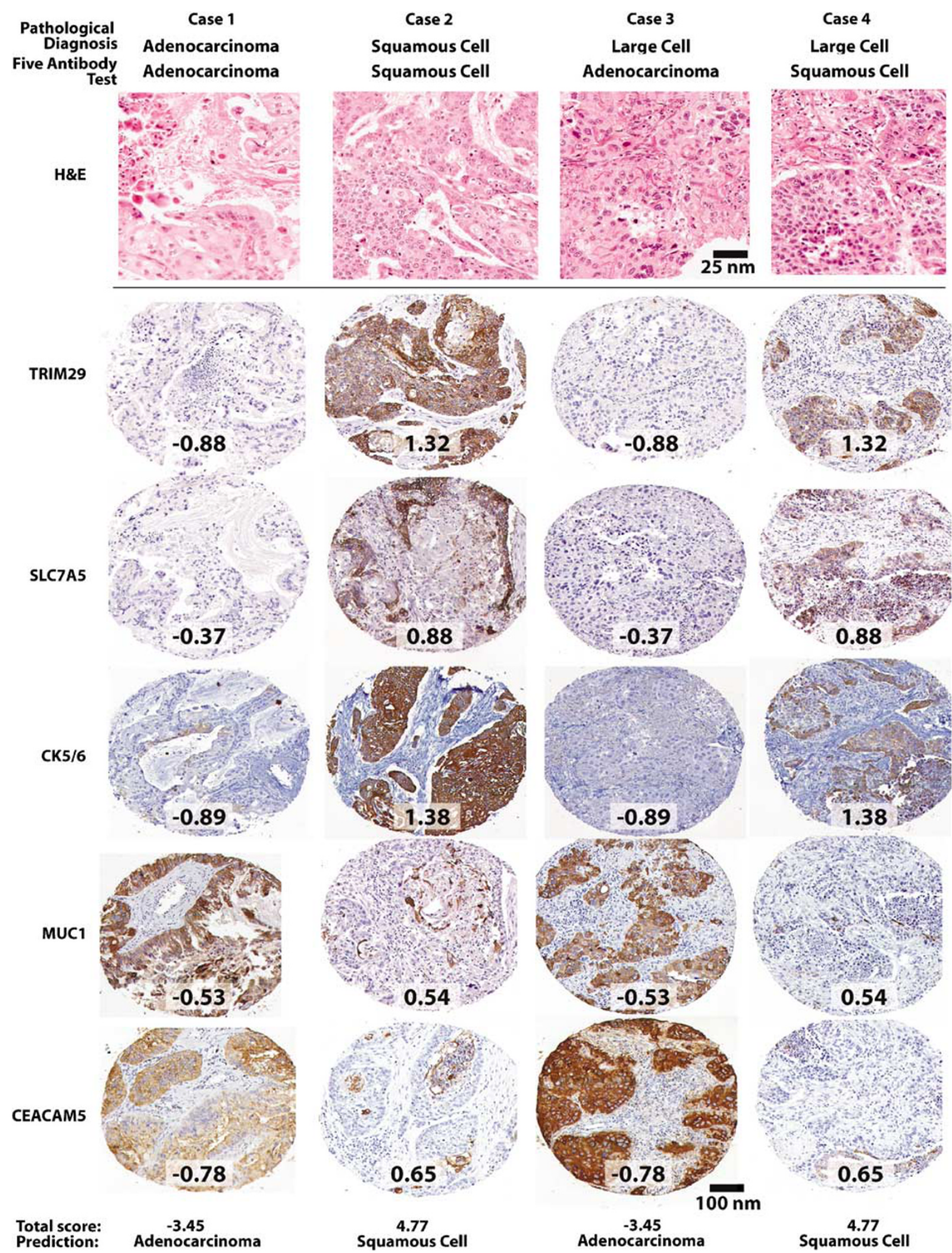

Figure 2 Representative cases stained with the five antisera. Four tumor samples stained with H\&E or the antisera comprising the fiveantibody test are shown with their respective morphological-based diagnoses. The score given to each case for each antibody in the fiveantibody test is given, as well as a cumulative score and predicted histological type. Note that the scale for the H\&E samples differs from that of the antibody-stained samples. 


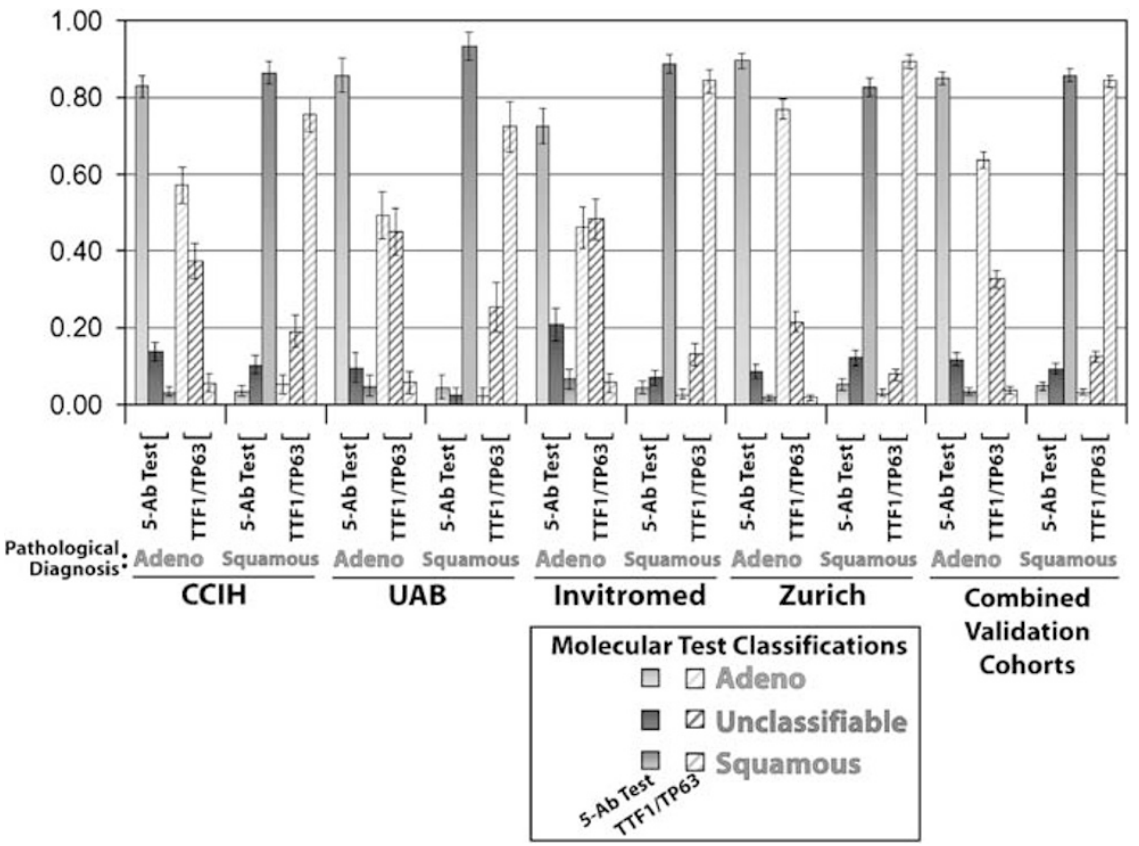

Figure 3 Classification of patient samples by the five-antibody test and TTF-1/TP63. The tumor samples from the four cohorts, which were classified as either squamous cell or adenocarcinoma by pathological diagnosis, are categorized on the $x$ axis, and the fraction of each classified by the two tests as 'squamous cell', 'unclassifiable', or 'adenocarcinoma' is shown. The error bars represent one standard error of the proportion. Classification by the five-antibody test is shown by the solid bars, and the two-antibody test by hatched bars. 'Combined validation cohorts' for the five-antibody test are the UAB, Invitromed, and Zurich populations; and for TTF-1/TP63 are the $\mathrm{CCIH}, \mathrm{UAB}$, Invitromed, and Zurich populations.

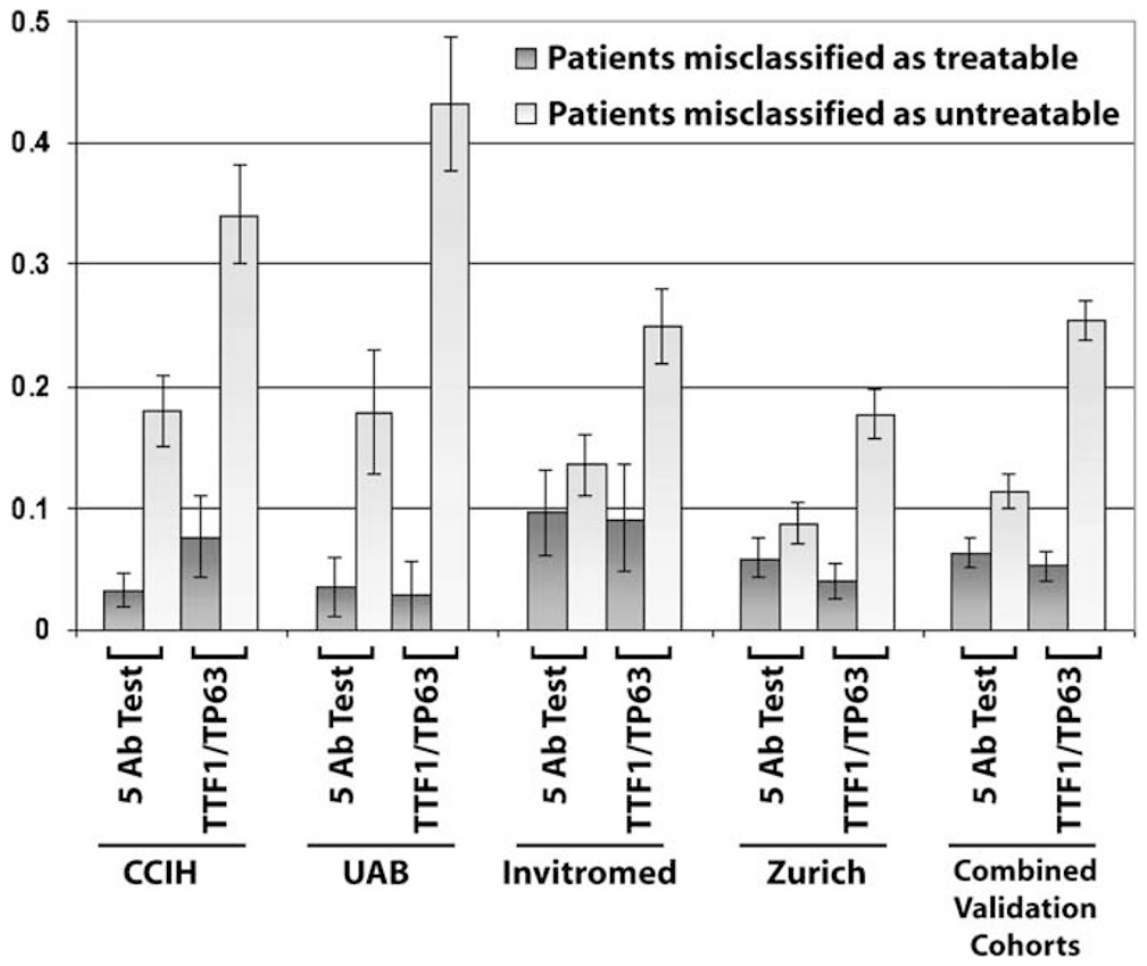

Figure 4 Utility of the five- and two-antibody classifiers in directing patient therapy choices with bevacizumab based on their molecular classification. Patients who are classified as adenocarcinomas by the molecular tests, and therefore candidates for treatment, yet are diagnosed as squamous cell carcinoma by a pathologist through morphological assessment are designated as 'misclassified as treatable'. Patients who classified as squamous or unclassifiable by the tests, and therefore should be excluded from treatment, yet diagnosed as adenocarcinoma by a pathologist are designated as 'misclassified as untreatable'. The error bars represent one standard error of the proportion. 'Combined validation cohorts' for the five-antibody test are the UAB, Invitromed, and Zurich populations; and for TTF-1/TP63 are the CCIH, UAB, Invitromed, and Zurich populations. 


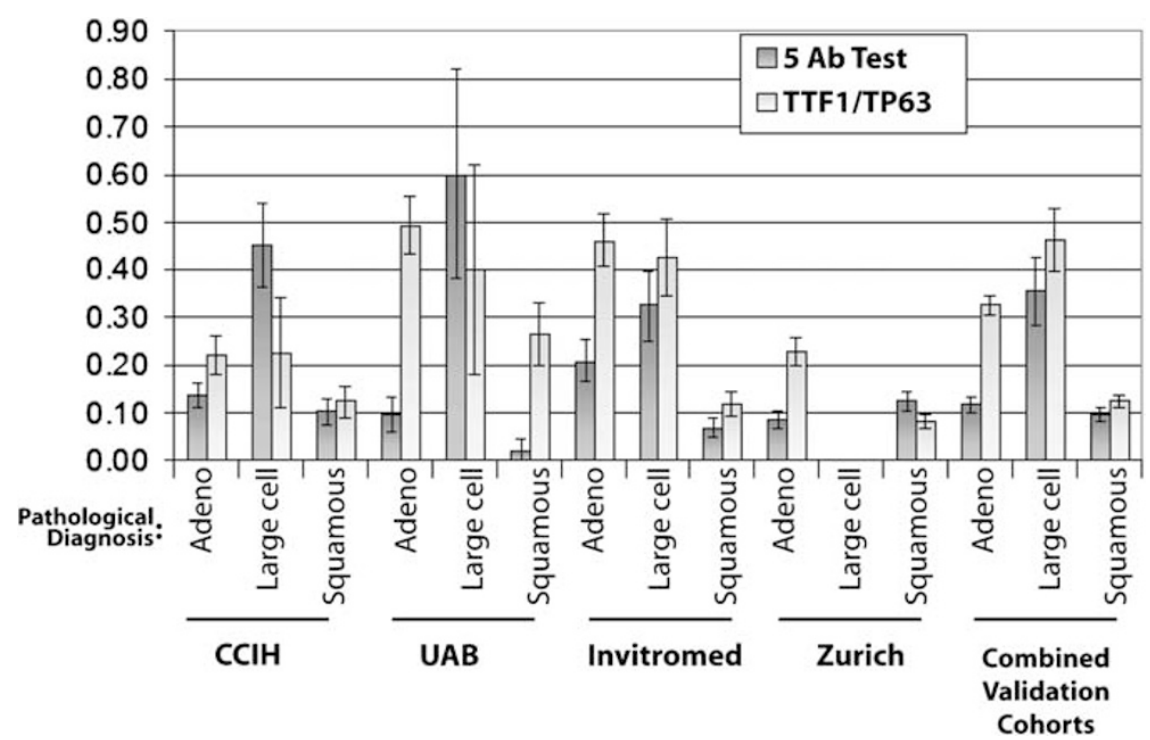

Figure 5 Distribution of samples unclassified by the antibody tests. The tumor samples from the four cohorts that were classified as either squamous cell, large cell, or adenocarcinoma by pathological diagnosis are categorized on the $x$ axis, and the fraction of each classified by the two tests as 'unclassifiable' is exhibited. The error bars represent one standard error of the proportion. Both molecular tests show a strikingly decreased ability to declare patients as either squamous or adenocarcinoma when the tumor had been diagnosed as large cell and TTF-1/TP63 has a much higher overall fraction of unclassifiable cases.

were able to give a definitive diagnosis and which were given either a pathological diagnosis of large cell, a variety of other descriptive diagnoses besides adenocarcinoma or squamous cell, or for which there were insufficient morphological clues to allow the pathologist to assign a diagnosis beyond the exclusion of small cell carcinoma, the five-antibody test and TTF-1/TP63 agreed 94\% of the time (Table 3) when each was able to make an unambiguous diagnosis. Both immunohistochemical tests showed a strikingly decreased ability to identify patients as either adenocarcinoma or squamous cell when the tumor had been morphologically diagnosed as large cell (Figure 5); $33-60 \% \quad(36 \%$ in the combined validation populations) for the five-antibody test, as opposed to $9-21 \%$ for those diagnosed as adenocarcinoma ( $12 \%$ for the combined population), and $2-$ $13 \%$ for those diagnosed as squamous cell carcinoma ( $10 \%$ for the combined population). In contrast, $59 \%$ of tumors morphologically classified as large cell showed an immunophenotype consistent with a diagnosis of either adenocarcinoma or squamous cell carcinomas with the five-antibody test and $56 \%$ with TTF-1/TP63 (Table 3). When both tests were able to make a diagnostic call, the results between the five-antibody test and TTF-1/TP63 were consistent for $96 \%$ of these cases interpreted as large cell by morphology.

\section{Discussion}

Lung carcinoma is routinely subclassified into the histological variants of small cell carcinoma, adenocarcinoma, squamous cell carcinoma, and large cell carcinoma based on morphological features. ${ }^{1}$ These subclasses differ not only in their presentation in different regions of the lung, but in outcomes as well, with large cell and adenocarcinomas tending to worse outcomes than squamous cell carcinoma. Despite these differences, histological type has not traditionally been an important factor in selecting treatment among non-small-cell lung carcinomas. ${ }^{9-11}$ Reflecting this historical lack of established clinical utility for lung carcinoma classification, there is currently no standard molecular-based classifier for histological type diagnosis. A need for standardized classification of lung carcinoma is growing as novel molecularly targeted therapies have shown different activities in histological subclasses. Furthermore, the importance of molecular aids to diagnosis has increased as needle biopsies specimens are becoming common in diagnosis of advanced disease. Owing to the limited tissue sample, there is a relatively high rate of morphological misdiagnosis in needle biopsy samples compared to surgical specimens. ${ }^{20}$

The results presented in this paper, comparing a novel five-antibody immunohistochemistry test and a more standard two-antibody test both performed on $600 \mu \mathrm{M}$ tissue array cores, to the reference 'gold standard'-morphological diagnosis on surgical resection samples, simulate the problem of diagnosis on small samples. The diagnostic specificity of both tests was validated on three independent cohorts with a combined population of over a thousand patient samples. These studies demonstrated a low misclassification rate for both the five-antibody test and TTF-1/TP63, very high agreement between the two tests when both made a diagnosis, and a much 
Table 3 Agreement analysis of tumor samples by the antibody tests

Five-antibody test

\begin{tabular}{|c|c|c|c|c|}
\hline \multirow[b]{2}{*}{ Pathologist diagnosis } & \multirow[b]{2}{*}{$T T F-1 / T P 63$} & \\
\hline & & Adenocarcinoma & Unclassifiable & Squamous cell \\
\hline \multirow{3}{*}{ Adenocarcinoma } & Adenocarcinoma & 288 & 22 & 0 \\
\hline & Unclassifiable & 113 & 33 & 3 \\
\hline & Squamous cell & 1 & 4 & 13 \\
\hline \multirow{3}{*}{ Large cell } & Adenocarcinoma & 7 & 3 & 0 \\
\hline & Unclassifiable & 4 & 16 & 4 \\
\hline & Squamous cell & 1 & 3 & 16 \\
\hline \multirow{3}{*}{ Other NSCLC } & Adenocarcinoma & 11 & 3 & 1 \\
\hline & Unclassifiable & 7 & 22 & 2 \\
\hline & Squamous cell & 2 & 2 & 24 \\
\hline \multirow{3}{*}{ Squamous cell } & Adenocarcinoma & 8 & 8 & 0 \\
\hline & Unclassifiable & 10 & 11 & 37 \\
\hline & Squamous cell & 6 & 30 & 421 \\
\hline
\end{tabular}

Abbreviation: NSCLC, non-small cell lung carcinoma.

The tumor samples from the four cohorts that were classified as either squamous cell, large cell, adenocarcinoma or 'other' (lung carcinoma not otherwise specified, mucoepidermoid, etc) by pathological diagnosis are categorized by rows, and the number of each classified by the two tests as 'squamous cell', 'unclassifiable', or 'adenocarcinoma' is exhibited.

lower rate of failure to classify samples for the fiveantibody test, enabling a diagnosis for a larger number of patients. This likely reflects that using these five markers allows a greater sampling of the physiological diversity within the biological subclasses, while still maintaining the specificity of class assignment and using few enough stains to be practical in a standard clinical laboratory setting. The concordance between diagnoses demonstrated in this study between two independent immunohistochemical-based tests and their high concordance with morphological diagnosis performed on surgical specimens affirms the robust nature of the biological classification and the tests.

The five antisera used by the test belong to a variety of biochemical or differentiation pathways, yet all have been seen to exhibit differential expression in tumor tissues. SLC7A5 is part of a two-protein complex with SLC3A2, the heavy chain of a neutral amino-acid transporter implicated in nutrient transport at the blood-brain barrier and has been noted to be differentially expressed between adeno- and squamous cell lung carcinomas. ${ }^{29,30}$ CEACAM5 is a glycosylphosphatidylinositol-anchored protein member of the immunoglobulin supergene family and has been seen to be differentially expressed among lung histological types. ${ }^{31}$ TRIM29 contains multiple zinc-finger motifs and a leucine zipper motif and thus may act as a transcriptional regulatory factor. Though little is known of its function in lung carcinogenesis, it has been noted as being differentially expressed between pancreatic ductal and chronic pancreatitis and is a consistent member of 'basal' breast carcinoma classifiers identified in gene expression profiling experiments. ${ }^{32}$ MUC1 is a member of the mucin family and encodes a membrane-bound, heavily glycosylated protein. The protein contains a variable number of tandem repeats (from 25 to 125 repeats) in its extracellular domain; the large MUC1 allele has been shown to be significantly associated with lung adenocarcinoma but not with squamous cell carcinoma. ${ }^{33}$ CK5/6 recognizes cytokeratin 5 and 6, type II cytokeratins who have been shown to be strongly differentially expressed in lung carcinoma subtypes and is also a marker of basal breast cancer. $^{21}$

Beyond its use in the pathology lab to augment morphology in determining histological type, molecular-based classifiers such as this five-antibody test have the potential to make farther reaching changes in lung carcinoma clinical practice. For example, the pathophysiology of the risk of hemorrhage with bevacizumab administration is unclear; however, the association with squamous cell differentiation is well established. There remains a small risk of hemorrhage in patients with presumed adeno differentiation, ${ }^{19}$ and given the uncertainty in making a diagnosis on needle biopsy tissue, it is possible that misclassification of tumor phenotype might account for some of these cases. For the purpose of confirming a diagnosis of adenocarcinoma and therefore identifying candidates for bevacizumab treatment, the misclassification rate of the fiveantibody test and TTF-1/TP63 as 'non-squamous' (ie as candidates for treatment) was similar (6 and $5 \%$, respectively, with $95 \%$ CI of $4-9$ and $3-8 \%$ ). However, the five-antibody test had a much lower rate of unclassified cases ( 11 vs $25 \%$, 95\% CI of 9-14 and $22-29 \%$ ) and therefore was able to identify a significantly larger number of adenocarcinoma patients who were candidates for targeted therapy.

The use of the five-antibody test might diminish the classification error rate even in cases where 
there is sufficient tissue for a morphological diagnosis, resulting in decreased risk to the patient. In support of this, the two molecular tests used in this analysis agreed on over $99.5 \%$ of the morphologically diagnosed adenocarcinoma samples when each test was able to make an unambiguous diagnosis. In total, $4.3 \%$ of the samples diagnosed by morphology as adenocarcinoma were reclassified as squamous cell carcinomas by both molecular tests (Table 3). This suggests that molecular features may be an invaluable aid in increasing confidence in determining patient class for therapy selection. A similar impact on the response rate of the EGFR inhibitors gefitinib and erlotinib might be anticipated by improving histological classification.

These studies have also demonstrated the potential for the use of the five-antibody test in assignment of a subset of morphological large cell cases to adeno- or squamous cell differentiation classes. Classification of large cell carcinoma has been traditionally defined by negative criteria, based on lack of squamous, glandular, or small cell features. Large cell carcinomas are thus, by definition, poorly differentiated tumors with a diagnosis of no more than $10 \%$ of cells consistent with squamous or adenocarcinoma differentiation in the sample. This subgroup is typically small (usually in the order of $10 \%$ of a cohort) and no specific response to therapy had been associated with it. Recently there has been an effort to better define this group of tumors by using molecular and genetic features as well as morphological features ${ }^{34}$ and has discriminated two new tumor entities within morphological large cell carcinoma, the neuroendocrine carcinomas ${ }^{35}$ and basaloid carcinomas ${ }^{36}$ both of which have a poor prognosis. Both TTF-1/TP63 and the five-antibody test identify an increased fraction of unclassifiable samples when the tumor had been morphologically diagnosed as large cell (Figure 5) consistent with an undifferentiated phenotype or with molecular features distinct from adenocarcinoma or squamous cell carcinoma. Interestingly, when both tests were able to make an unambiguous diagnostic call among the pathologically defined large cell cases, the results between the five-antibody test and TTF-1/ TP63 were consistent for $96 \%$ of these cases, suggesting that the molecular features of this subset of samples are more similar to squamous or adenocarcinomas, and may be properly classified as containing differentiated features. Similarly, for all cases where pathologists either declined to make a histological call, or where diagnosis of distinctive morphology was noted (mucoepidermoid, basaloid, etc), these tests agreed $92 \%$ of the time (Table 3 ). The appropriate management of large cell cases or other rare morphological types, reclassified as adeno- or squamous cell by molecular analysis with reliable, validated assays like this five-antibody test will need to be explored in larger clinical trials with appropriate clinical outcome data.
In the studies presented in this paper we show that a five-antibody test is able to reproducibly distinguish adenocarcinoma and squamous cell carcinoma lung carcinoma tumors and is superior to two biomarkers targeting TTF-1 and TP63 in correctly classifying patients. These studies have also demonstrated the potential of a five-antibody test in assignment of a subset of morphological large cell cases to adenocarcinoma or squamous cell classes. Furthermore, our findings suggest that this test could improve patient stratification as well as increase the number of patients deemed eligible for emerging targeted therapies by increasing the sensitivity and confidence with which histological type diagnosis can be established on needle biopsies. In this regard, prospectively defined clinical trials assessing the five-antibody test as an aid to clinical stratification might increase the number of patients deemed candidates for emerging targeted therapies. Most importantly, these results suggest the fiveantibody test may have an immediate role to pathologists in complimenting the morphological assessment of lung carcinoma histological types. The increased use of needle biopsies for diagnosis of late stage disease, and the consequent diminished amount of tissue available for interpretation, has created a pressing need for such a molecular-based classifier in the clinic.

\section{Disclosure/conflict of interest}

The authors Brian Z Ring, Robert S Seitz, Rodney A Beck, William J Shasteen, and Douglas T Ross are employed by and have stock interest in Applied Genomics Inc. Marshall $\mathrm{T}$ Schreeder has a stock interest in Applied Genomics Inc.

\section{References}

1 Beasley MB, Brambilla E, Travis WD. The 2004 World Health Organization classification of lung tumors. Semin Roentgenol 2005;40:90-97.

2 Chen HY, Yu SL, Chen CH, et al. A five-gene signature and clinical outcome in non-small-cell lung cancer. $\mathrm{N}$ Engl J Med 2007;356:11-20.

3 Garber ME, Troyanskaya OG, Schluens K, et al. Diversity of gene expression in adenocarcinoma of the lung. Proc Natl Acad Sci USA 2001;98:1378413789.

4 Liu H, Kho AT, Kohane IS, et al. Predicting survival within the lung cancer histopathological hierarchy using a multi-scale genomic model of development. PLoS Med 2006;3:e232.

$5 \mathrm{Lu} \mathrm{Y,} \mathrm{Lemon} \mathrm{W,} \mathrm{Liu} \mathrm{PY,} \mathrm{et} \mathrm{al.} \mathrm{A} \mathrm{gene} \mathrm{expression}$ signature predicts survival of patients with stage I nonsmall cell lung cancer. PLoS Med 2006;3:e467.

6 Meyerson M, Franklin WA, Kelley MJ. Molecular classification and molecular genetics of human lung cancers. Semin Oncol 2004;31(1 Suppl 1):4-19.

7 Sun S, Schiller JH, Spinola M, et al. New molecularly targeted therapies for lung cancer. J Clin Invest 2007;117:2740-2750. 
8 Rossi G, Marchioni A, Sartori G, et al. Histotype in non-small cell lung cancer therapy and staging: the emerging role of an old and underrated factor. Curr Resp Med Rev 2007;3:69-77.

9 Pfister DG, Johnson DH, Azzoli CG, et al. American Society of Clinical Oncology treatment of unresectable non-small-cell lung cancer guideline: update 2003. J Clin Oncol 2004;22:330-353.

10 Albain KS, Crowley JJ, LeBlanc M, et al. Survival determinants in extensive-stage non-small-cell lung cancer: the Southwest Oncology Group experience. J Clin Oncol 1991;9:1618-1626.

11 Schiller JH, Harrington D, Belani CP, et al. Comparison of four chemotherapy regimens for advanced nonsmall-cell lung cancer. N Engl J Med 2002;346:92-98.

12 Lynch TJ, Bell DW, Sordella R, et al. Activating mutations in the epidermal growth factor receptor underlying responsiveness of non-small-cell lung cancer to gefitinib. N Engl J Med 2004;350:2129-2139.

13 Paez JG, Janne PA, Lee JC, et al. EGFR mutations in lung cancer: correlation with clinical response to gefitinib therapy. Science 2004;304:1497-1500.

14 Shepherd FA, Rodrigues Pereira J, Ciuleanu T, et al. Erlotinib in previously treated non-small-cell lung cancer. N Engl J Med 2005;353:123-132.

15 Georgoulias V, Papadakis E, Alexopoulos A, et al. Platinum-based and non-platinum-based chemotherapy in advanced non-small-cell lung cancer: a randomised multicentre trial. Lancet 2001;357:1478-1484.

16 Scagliotti GV, Parikh P, von Pawel J, et al. Phase III study comparing cisplatin plus gemcitabine with cisplatin plus pemetrexed in chemotherapy-naive patients with advanced-stage non-small-cell lung cancer. J Clin Oncol 2008;26:3543-3551.

17 Herbst RS, Johnson DH, Mininberg E, et al. Phase I/II trial evaluating the anti-vascular endothelial growth factor monoclonal antibody bevacizumab in combination with the HER-1/epidermal growth factor receptor tyrosine kinase inhibitor erlotinib for patients with recurrent non-small-cell lung cancer. J Clin Oncol 2005;23:2544-2555.

18 Johnson DH, Fehrenbacher L, Novotny WF, et al. Randomized phase II trial comparing bevacizumab plus carboplatin and paclitaxel with carboplatin and paclitaxel alone in previously untreated locally advanced or metastatic non-small-cell lung cancer. J Clin Oncol 2004;22:2184-2191.

19 Sandler A, Gray R, Perry MC, et al. Paclitaxelcarboplatin alone or with bevacizumab for non-smallcell lung cancer. N Engl J Med 2006;355:2542-2550.

20 Edwards SL, Roberts C, McKean ME, et al. Preoperative histological classification of primary lung cancer: accuracy of diagnosis and use of the non-small cell category. J Clin Pathol 2000;53:537-540.

21 Kargi A, Gurel D, Tuna B. The diagnostic value of TTF1, CK 5/6, and p63 immunostaining in classification of lung carcinomas. Appl Immunohistochem Mol Morphol 2007;15:415-420.

$22 \mathrm{Wu}$ M, Szporn AH, Zhang D, et al. Cytology applications of p63 and TTF-1 immunostaining in differential diagnosis of lung cancers. Diagn Cytopathol 2005;33: 223-227.

$23 \mathrm{Wu} \mathrm{M}$, Wang $\mathrm{B}$, Gil J, et al. p63 and TTF-1 immunostaining. A useful marker panel for distinguishing small cell carcinoma of lung from poorly differentiated squamous cell carcinoma of lung. Am J Clin Pathol 2003;119:696-702.

$24 \mathrm{Au}$ NH, Gown AM, Cheang M, et al. P63 expression in lung carcinoma: a tissue microarray study of 408 cases. Appl Immunohistochem Mol Morphol 2004;12: 240-247.

25 Pelosi G, Fraggetta F, Pasini F, et al. Immunoreactivity for thyroid transcription factor-1 in stage I non-small cell carcinomas of the lung. Am J Surg Pathol 2001;25:363-372.

26 Tan D, Li Q, Deeb G, et al. Thyroid transcription factor1 expression prevalence and its clinical implications in non-small cell lung cancer: a high-throughput tissue microarray and immunohistochemistry study. Hum Pathol 2003;34:597-604.

27 Ring BZ, Seitz RS, Beck R, et al. Novel prognostic immunohistochemical biomarker panel for estrogen receptor-positive breast cancer. J Clin Oncol 2006;24:3039-3047.

28 Mallows CL. Some comments on Cp. Technometrics 1973;15:661-675.

29 Kaira K, Oriuchi N, Imai H, et al. Prognostic significance of L-type amino acid transporter 1 expression in resectable stage I-III nonsmall cell lung cancer. $\mathrm{Br} \mathrm{J}$ Cancer 2008;98:742-748.

30 Kido Y, Tamai I, Uchino $\mathrm{H}$, et al. Molecular and functional identification of large neutral amino acid transporters LAT1 and LAT2 and their pharmacological relevance at the blood-brain barrier. J Pharm Pharmacol 2001;53:497-503.

31 Blumenthal RD, Leon E, Hansen HJ, et al. Expression patterns of CEACAM5 and CEACAM6 in primary and metastatic cancers. BMC Cancer 2007;7:2.

32 Chen Y, Zheng B, Robbins DH, et al. Accurate discrimination of pancreatic ductal adenocarcinoma and chronic pancreatitis using multimarker expression data and samples obtained by minimally invasive fine needle aspiration. Int J Cancer 2007;120: 1511-1517.

33 Mitsuta K, Yokoyama A, Kondo K, et al. Polymorphism of the MUC1 mucin gene is associated with susceptibility to lung adenocarcinoma and poor prognosis. Oncol Rep 2005;14:185-189.

34 Brambilla E, Travis WD, Colby TV, et al. The new World Health Organization classification of lung tumours. Eur Respir J 2001;18:1059-1068.

35 Travis WD, Linnoila RI, Tsokos MG, et al. Neuroendocrine tumors of the lung with proposed criteria for large-cell neuroendocrine carcinoma. An ultrastructural, immunohistochemical, and flow cytometric study of 35 cases. Am J Surg Pathol 1991;15:529-553.

36 Brambilla E, Moro D, Veale D, et al. Basal cell (basaloid) carcinoma of the lung: a new morphologic and phenotypic entity with separate prognostic significance. Hum Pathol 1992;23:993-1003. 